\title{
Tribalism and Local Structures in a Music and Video Installation
}

\author{
Theodoros Lotis
}

\author{
Department of Music, Ionian University \\ Corfu-Greece \\ Email: lotis@ionio.gr
}

\begin{abstract}
.
In a world of globalisation, where economic borders are suppressed whilst national and racial barriers are erected higher, the notions of tribalism and tribal behaviorism remain as dominant and indicative as always. Although tribes no longer exist, in the western world, these notions are nowadays related to groups, such as political or athletic associations, economic factions, trade-union organisations and professional guilds, religious teams, social networks on the Internet, e.t.c. These groups often have simple structures with few significant distinctions between their individuals. Tribalism describes the loyalties that individuals feel towards their group/tribe and the way these loyalties affect their behavior and their attitudes towards others. It also refers to the strong cultural identity that characterises oneself as a member of one group/tribe. These characteristics shape the tribal consciousness and loyalty to the tribal values and to the customs and beliefs of a tribal society. Each time a tribe is in danger of corruptive influences from external sources (other tribes), these values provide for strategies of defense. This paper describes the strategies and methodologies for creating and evolving local structures in a music and video installation. The installation consists of two videos with their unique sound track and a live video, which also contains a triggered sound. A microphone collects, transforms and feeds back sounds and noises generated by the visitors.
\end{abstract}

\section{Motivations and interactivity.}

In between our philosophical and scientific approach to our planet (global spaceearth) and the behavior in our everyday life places (habitable spaces-houses, offices, local environments), lies down a personal, intimate space, which is our observatory for both global and habitable spaces. This intimate space, inherent to all human beings and sculpted through centuries, is packed with knowledge, prejudices, limitations and prerequisites. This installation provides a space for action and contemplation of our intimacies. The visitors/unintentional performers, surrounded by projections of global and habitable spaces, investigate their intimate spaces in relation to the global and habitable spaces.

A level of interaction is needed in order to prompt the visitors and activate this investigation. Defining interactivity within a digital environment is a complex task since "...the term's meaning is constantly shifting" (Aarseth, 2003). In any case, the scope of this paper is not to define but to describe digital interactivity in a music and video installation. However, since "...digital technology can give artists the opportunity to transform visitors from passive viewers into active participants" (Blum, 2007), it is important to point out that digital interactivity often encourages the 
users to communicate with themselves through the medium. David Rokeby describes this process as follows:

The medium not only reflects back, but also refracts what it is given; what is returned is ourselves, transformed and processed. To the degree that the technology reflects ourselves back recognizably, it provides us with a self-image, a sense of self. To the degree that the technology transforms our image in the act of reflection, it provides us with a sense of the relation between this self and the experienced world. (1995.)

The interaction for the installation is provided by a stable camera, which detects the visitors' motion and by a stable microphone that captures the visitors' utterance. Both camera and microphone, collect, transform and feed back their input sources in form of refracted intimacies.

\section{Local structures.}

The installation runs in loops of $7 \frac{1 / 2}{2}$ minutes each. This temporal structure is controlled by a subpatch ('time') (fig. 6), which will be analysed later in the paper.

\subsection{The tribes.}

The fundamentals for creating and controlling the local structures derive from the application of basic tribal rules. The installation - whose mechanism has been developed in Max/MSP (image 1) - consists of four sound groups, which will be referred to as tribes (fig. 1).

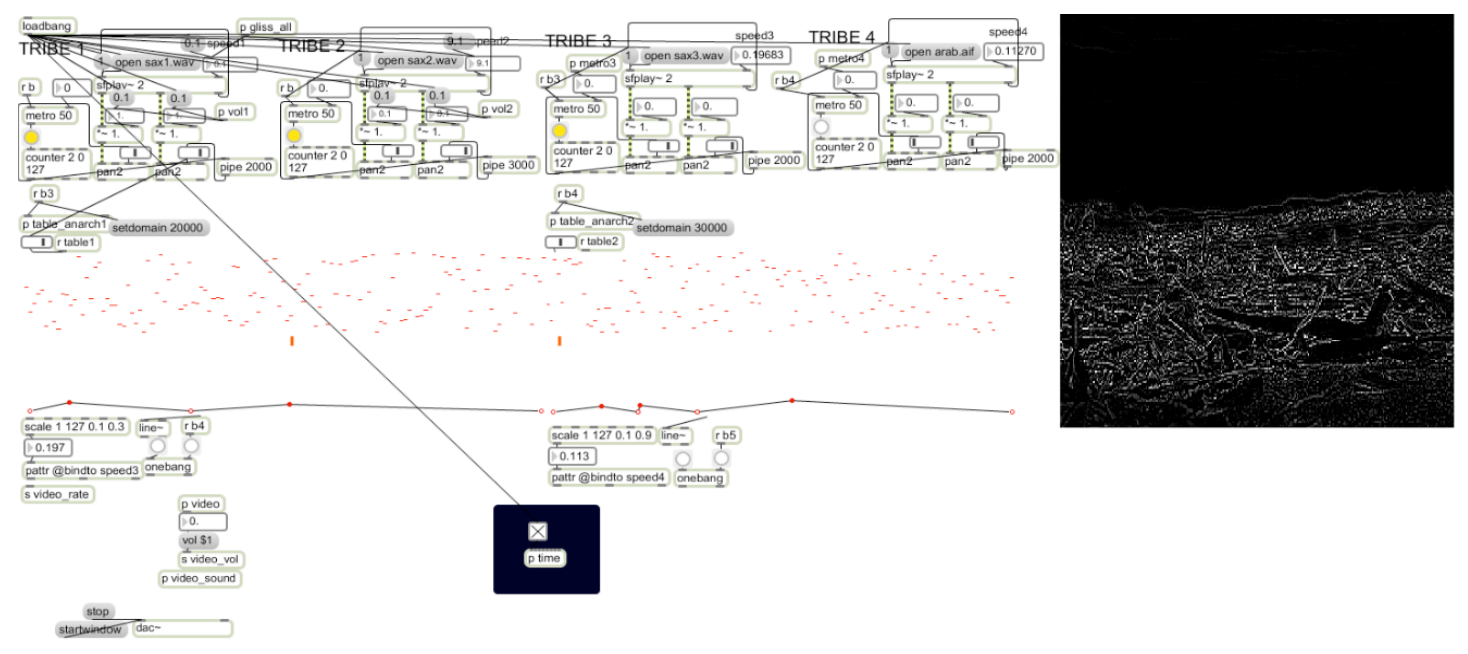

image 1: main patch for videos 1 and 2. 


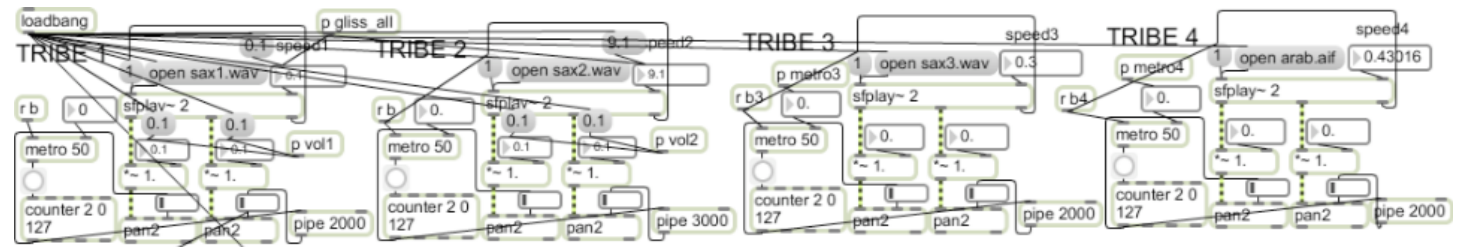

fig. 1: the tribes: 4 sound groups with local structures.

The first three tribes reproduce a recorded saxophone sound, whilst the fourth tribe reproduces a recorded voice. Each tribe has its own local/tribal rules and values, as well as mechanisms for defending its identity from external invasions. An external invasion - which is mainly done via sending and receiving bangs within subpatches is defined as an attempt of a tribe to degenerate the local sonic parameters of another tribe. The local rules and values of each tribe are related to sonic parameters, such as amplitude, playback speed and panoramic. For example, a tribe attacks another tribe by sending bangs, which infiltrate to its volume discontinuities and, often, glitches (fig. 2). The result is that the volume of the attacked tribe degenerates and lacks cohesion until it recuperates its local volume values.

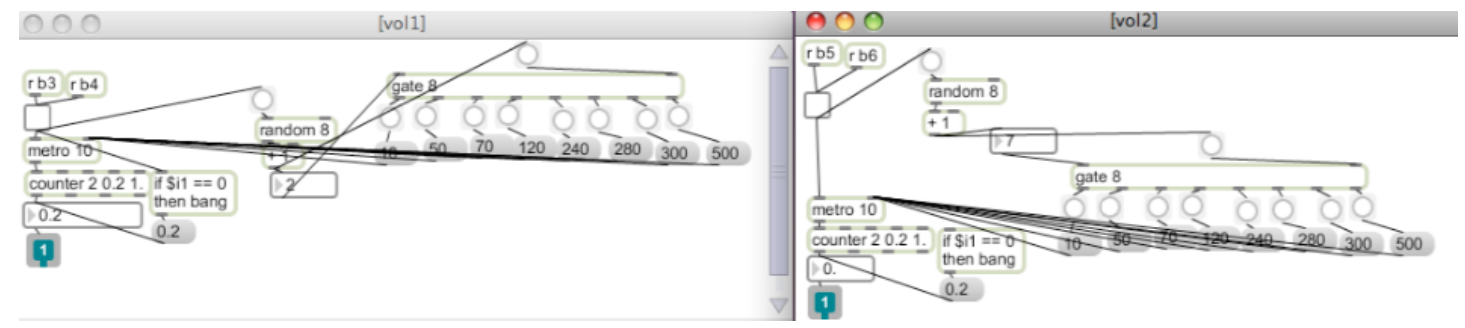

fig. 2: volume settings for tribes 1 and 2.

Using the same method, a tribe can also influence the panoramic's speed and position of the sound of another tribe (fig. 3).

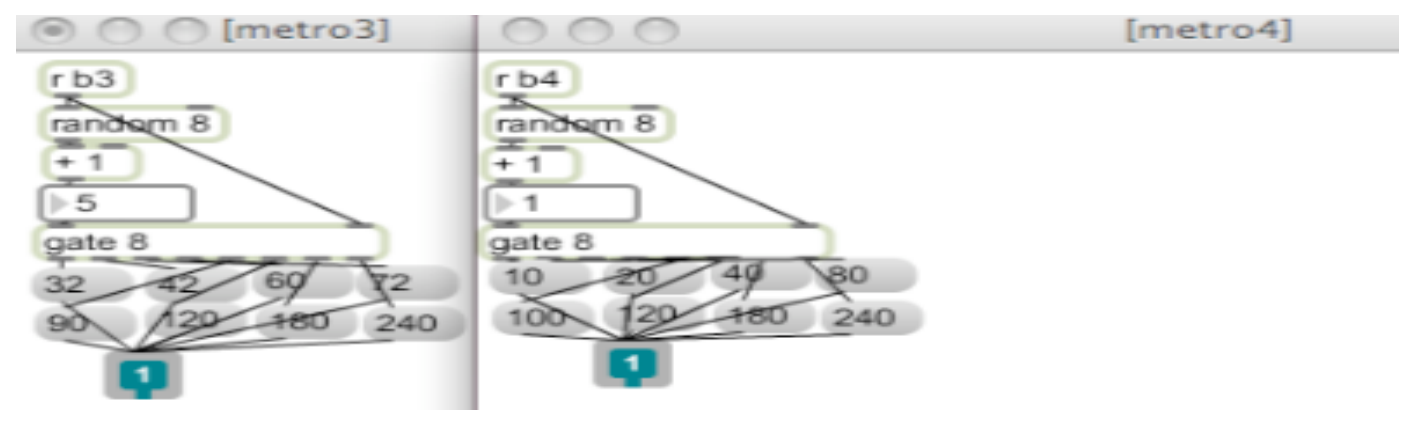

fig. 3: metronome settings for panoramic speed and position for tribes 3 and 4.

The most catastrophic attack a tribe can operate is via the subpatch called 'anarch'. By sending bangs, a tribe can alter the content range of the 2 itables (fig. 5), influencing thus the pitch, volume and playback speed of the sound in another tribe. However, due to the arguments of the 'random' objects, there are $20 \%$ possibilities of failure of the attempted attack (fig. 4). 


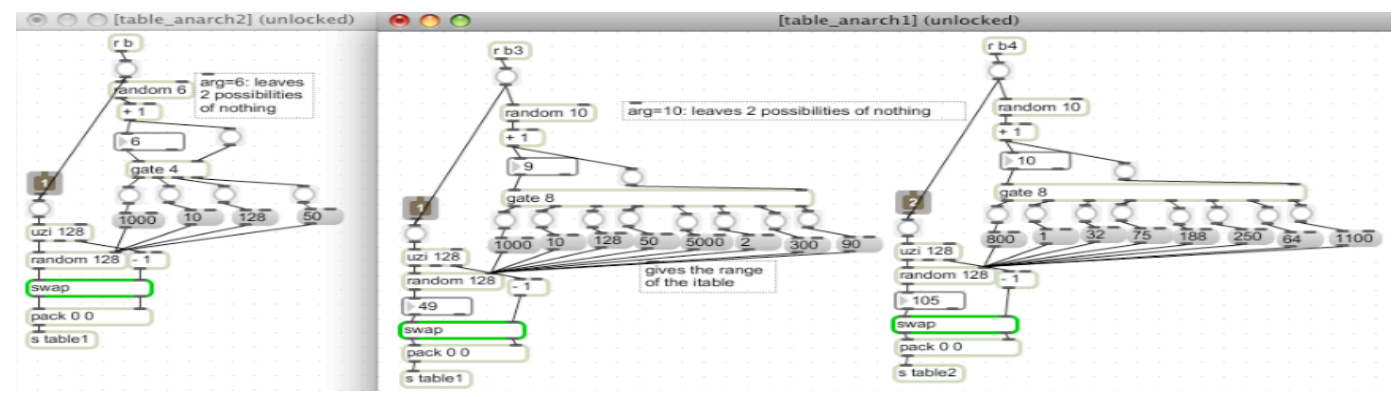

fig.4 : subpatches 'anarch 1' and 'anarch 2'.
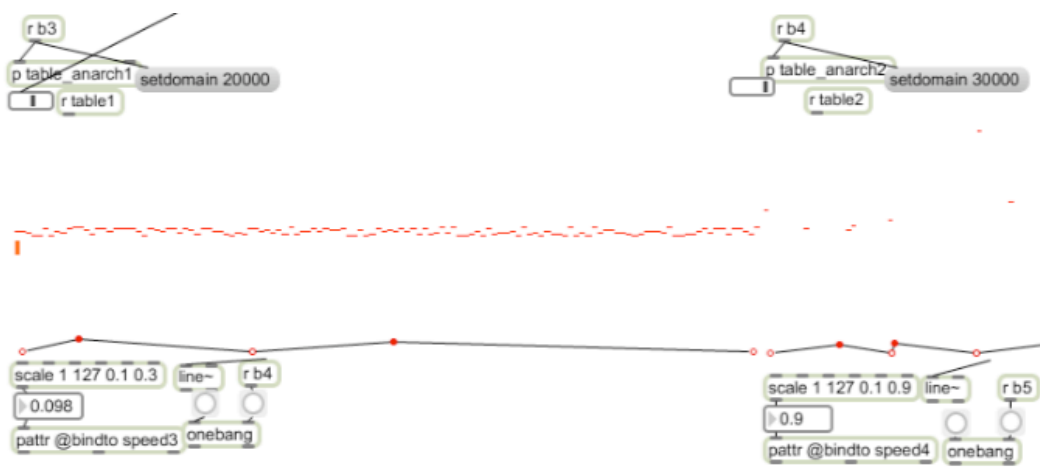

fig. 5: content range of the 2 itables influenced by the 'anarch' subpatches. The objects 'pattr' alter the playback speed of tribes 3 and 4 .

At the same time (fig. 5), numerical values and attributes, binded at the 'pattr' object, are also influencing the playback speed of the tribes 3 and 4 (speed 3 and 4 in fig. 1).

Thus, a self-evolving network of tribes is created, in which each tribe adjusts its structural and morphological development according to external influences and its own limitations.

\section{Global spaces: video 1 .}

Finally, the playback speed of the video is controlled by the playback speed of the sound in tribe 1. The video image passes through an edge detector filter. The perceived video images resemble the night city lights captured from a satellite source high in the sky. A night view from space, moving from city to city is established (vid. 1).

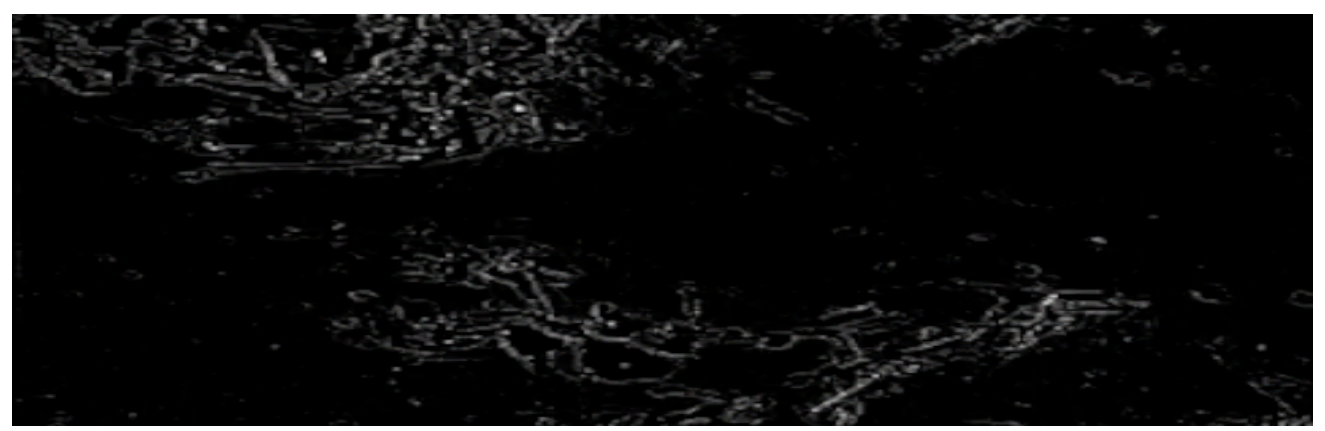

Vid. 1: excerpts from the first video: global spaces.

Theodoros Lotis 


\section{Habitable spaces: video 2.}

A second video is reproduced by another computer, which operates the same Max/MSP patch. This video also passes through the same edge detector filter, producing images which resemble architectural plans and designs (vid. 2). These images, which refer to our personal habitable spaces, contrast and converse with the distant and global images of the first video.

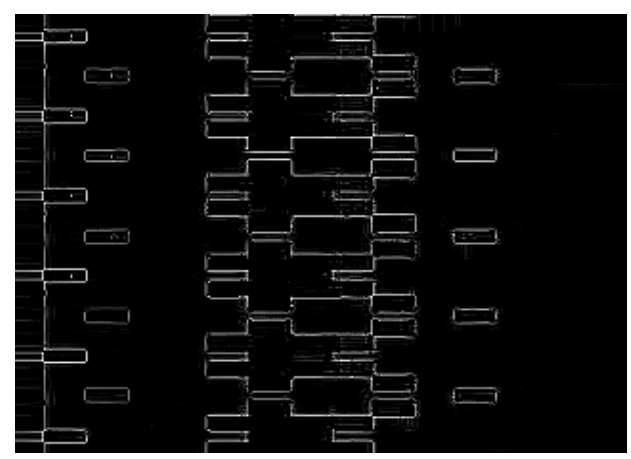

vid. 2: excerpts from the second video: habitable spaces.

\section{Crossing paths.}

\subsection{Intimate spaces: the live input video.}

Crossing the paths between global and habitable spaces, the live input video reserves a place for the human beings. This is the place for interaction between humans and the installation. The visitor's motion is captured by a video camera and feeded into a third computer which operates a different Max/MSP patch ${ }^{1}$. The patch sketches out moving figures of humans every time the camera detects motion. These moving figures correspond to the humans' motion and reveal fragmented hidden texts (image 2). In order to reveal the texts, the visitors of the installation have to move, triggering thus the process.

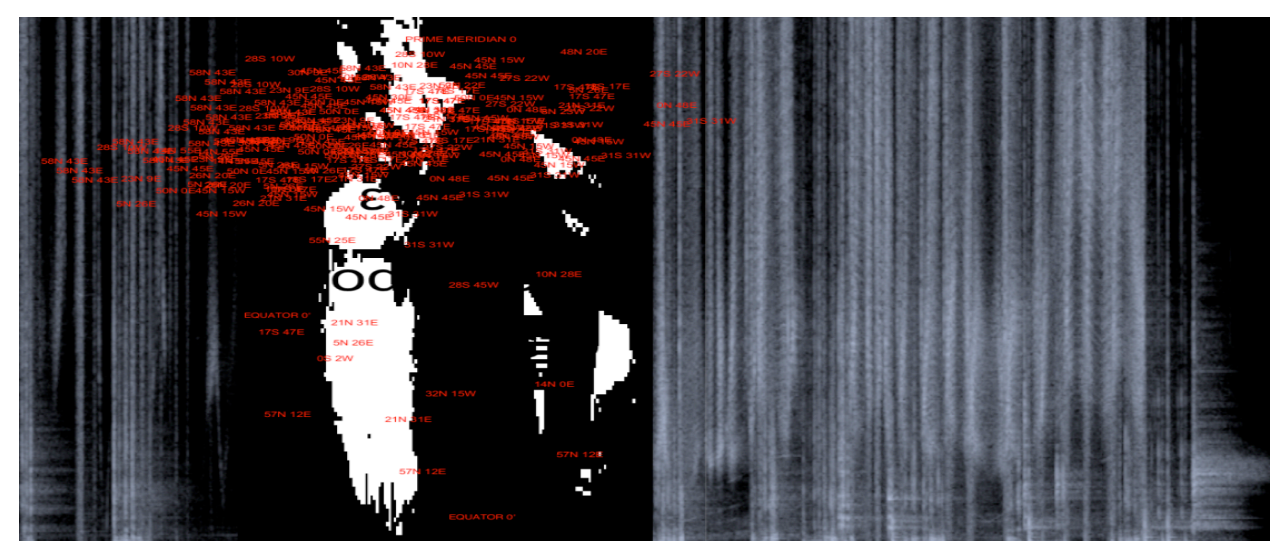

Image 2: figures of humans revealing hidden texts.

${ }^{1}$ Max/MSP patch by Andrew Benson. 


\subsection{Intimate spaces: the live input sound.}

The second level of interaction is produced by a Max/MSP patch with a microphone input (fig. 6). The sounds and the noises of the visitors are passed through a plug-in, which resonates parts of their spectra, before sending them to the loudspeakers.

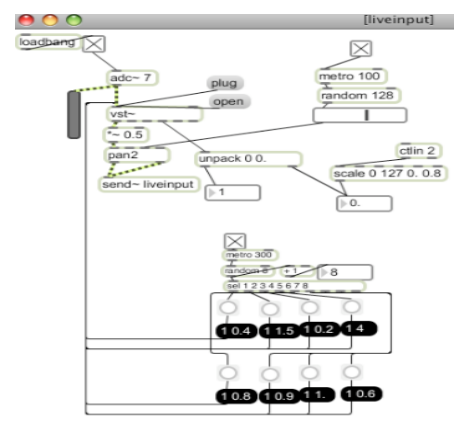

fig. 6: microphone input.

\section{High - level structure.}

At the highest structural level, a time machine controls the temporal development of the installation by producing cycles of predefined durations ( $71 / 2$ minutes each) (fig. 7). At the end of each loop, a subpatch ('gliss_all') is triggered resetting all parameters at new states (fig. 8). Through successive cycles, the tribes evolve influencing both music and image.
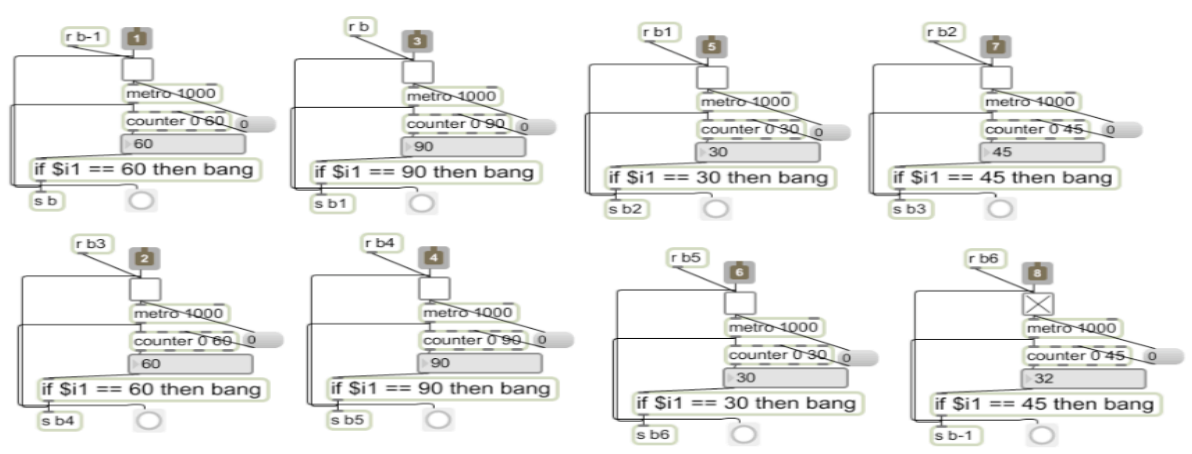

fig. 7: time control at high level: creating time loops.



fig. 8: resetting of all parameters every $7 \frac{1}{2}$ minutes. 


\section{Codetta.}

In order to experience at least one cycle, visitors should remain in the installation's space for a minimum of $7 \frac{1}{2}$ minutes. During this period of time, the visitor explores the projected and continuously changing global and habitable spaces. The visitor is invited to adjust a personal intimate space where his/her body motion and utterance unveils a refracted self; to cross the boundaries between the global and the local, the known and the unknown, the conscious faith and the unexpected result; to make the acquaintance of an undiscovered self via fragmented digitized images.

\section{References*.}

Aarseth, Espen. (2003). We all want to change the world: the ideology of innovation in digital media. Cambridge: MIT Press.

Augé, Marc. (1994). Pour une anthropologie des mondes contemporains. Paris: Aubier.

Blum, Frank. (2007). Digital interactive installations. Berlin: VDM Verlag Dr. Müller.

Heidegger, Martin. (1954). Bauen Wohnen Denken. In "Vorträge und Aufsätze". Gunter Neske.

Layton, Robert. (1991). The anthropology of art. Cambridge University Press.

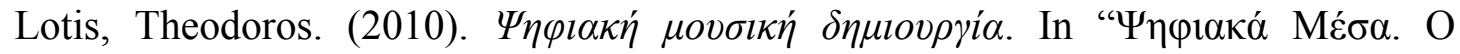

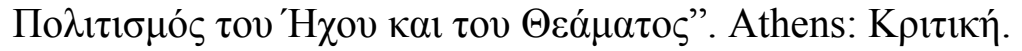

Mumford, Lewis. (1974). The Myth of the machine. Techniques and human development. Mariner Books.

Panofsky, Erwin. (1972). Studies in Iconology: Humanistic Themes in the Art of the Renaissance. Westview Press.

Rokeby, David. (1995). Transforming mirrors: subjectivity and control in interactive media. In "Critical Issues in Electronic Media". Ed. By Penny Simon. New York: SUNY Press.

* Additional information including the installation's setup plan and video and sound excerpts can be found at: http://www.theodorelotis.com 